\title{
Changes in Psychological Symptoms and Heart Rate Variability During EMDR Treatment: A Case Series of Subthreshold PTSD
}

\author{
Alessandra Frustaci \\ Institute of Psychiatry and Psychology, Catholic University of the Sacred Heart, Rome, Italy \\ Gaetano A. Lanza \\ Institute of Cardiology, Catholic University of the Sacred Heart, Rome, Italy \\ Isabel Fernandez \\ Psychotraumatology Research Centre and Italian EMDR Association, Milan, Italy \\ Massimo di Giannantonio \\ University “G. d'Annunzio," Chieti, Italy \\ Gino Pozzi \\ Institute of Psychiatry and Psychology, Catholic University of the Sacred Heart, Rome, Italy
}

\begin{abstract}
Elevated psychophysiological parameters and heightened physiological reactivity to trauma-related cues are acquired changes following trauma exposure. Measuring improvement in these variables is an appropriate evaluation of outcome in treatment studies. Heart Rate Variability (HRV) is a computerized measure of physiological responsivity derived from Holter ECG recording. Four female outpatients with persistent post-traumatic symptoms and personal impairment following "small t" trauma exposure underwent a course of EMDR treatment and were assessed at baseline, end of treatment, day 30 and day 90 of followup, using self-report symptom scales and 90-min Holter ECG recordings. Symptom scores decreased between baseline and end of treatment, with improvement maintained at follow-up. Several HRV measures changed favorably in different recording intervals. HRV is a feasible and sensitive method to measure physiological changes in the treatment of individuals distressed by "small t" trauma. Further investigation is advisable to expand these preliminary data.
\end{abstract}

Keywords: trauma; EMDR; IES; HRV; case series

$\mathbf{S}$ ymptoms following traumatic exposure include both psychological (e.g., intrusive recollection, re-experiencing, avoidance, affective detachment) and physiological (e.g., hyper-arousal, abnormal reactivity to cues, exaggerated startle response) alterations. Although clinical studies often evaluate only self-reported symptoms (Orr, McNally, Rosen, \& Shalev, 2004; Wilhelm \& Roth, 2001), elevated psychophysiological baseline scores and heightened physiological reactivity to trauma-related cues are typical features of posttraumatic stress disorder (PTSD) which can be objectively measured through cardiac parameters (Keane et al., 1998). Research has shown that elevated physiological characteristics are modifications that are acquired post trauma, and not pre-event risk factors for PTSD (Buckley \& Kaloupek, 2001; Pitman et al., 2006). Therefore, physiological measures are recommended to evaluate both the process of spontaneous recovery and the efficacy of therapies, independent from self-reported psychological symptoms (Orr et al., 2003; Orr \& Roth, 2000).

\section{Measurement of Physiological Symptoms}

The efficacy of EMDR in chronic PTSD has been established in a large number of studies, as summarized by Bisson et al. (2007). However, only a few investigators have used physiological measures as process 
and/or outcome variables. These included heart rate, skin temperature and conductance, electromyography, blood oxygen saturation, and respiratory carbon dioxide levels (Carlson, Chemtob, Rusnak, Hedlund, \& Moraoka, 1998; Elofsson, von Schèele, Theorell, \& Söndergaard, 2008; Montgomery \& Ayllon, 1994; Rogers et al., 1999; Wilson, Silver, Covi, \& Foster, 1996) and heart rate variability (Elofsson et al., 2008; Sack, Lempa, \& Lamprecht, 2007; Sack, Lempa, Steinmetz, Lamprecht, \& Hofmann, 2008).

In clinical practice a large proportion of civilian clients may show significant levels of PTSD symptoms even though they not have experienced a distressful condition that meets the Diagnostic and Statistical Manual of Mental Disorders 4th ed., text revision (DSMIV-TR; American Psychiatric Association [APA], 2000) PTSD diagnostic criteria for classification as a traumatic event (Gold, Marx, Soler-Baillo, \& Sloan, 2005; Mol et al., 2005; Van Hooff, McFarlane, Baur, Abraham, \& Barnes, 2009). Shapiro (2001) has labeled such events "small $t$ " traumas. These clients may request and benefit from an effective trauma treatment, such as EMDR (Cvetek, 2008). To our knowledge, no systematic data exist about the treatment modification of psychophysiological parameters in trauma-exposed subjects with subthreshold posttraumatic symptoms.

\section{Heart Rate Variability}

Heart Rate Variability (HRV) is the variation in the time intervals between consecutive normal heart beats, which is measured as the interval between consecutive $\mathrm{R}$ wave peaks on the electrocardiogram (R-R). The computerized elaboration of a continuous electrocardiogram (ECG) includes time-domain and frequency-domain analyses of HRV. Time-domain methods make use of simple statistical analyses to measure the amount of variability present in a definite time period. Frequency-domain methods analyze the R-R interval series using the mathematical techniques of fast Fourier transformation or autoregression analysis to quantify the amount of cyclical variations present at different frequencies (Pumprla, Howorka, Groves, Chester, \& Nolan, 2002).

The measure of HRV is a highly sensitive, reliable and noninvasive tool for quantitative assessment of cardiovascular autonomic regulatory responses; especially, an increased cardiac vagal tone is associated with elevated HRV (Stein, Bosner, Kleiger, \& Conger, 1994). Vagal control is more efficient than sympathetic control in modulating rapid changes in heart rate, due to the superior frequency response characteristics of cholinergic over adrenergic neurotransmitter systems (Saul, 1990). The degree of vagal control is a major determinant of responsivity and sensitivity of heart rate to changing environmental demands (Goldberger, 1991). So, high vagal tone is regarded as a marker for individual "flexibility" in various physiological and psychological conditions. Reductions in the responses of vagal autonomic function to a wide range of physiological stimuli are associated with a lack of adaptive variability in behavioral and cognitive functioning, and with poor health outcomes (Friedman \& Thayer, 1998).

HRV has been used to gauge the physiological alteration in a number of psychiatricillnesses (Agelink, Boz, Ullrich, \& Andrich, 2002; Boettger et al., 2006), including the effects of trauma-related cues in PTSD (Cohen et al., 2000a). Overall, altered vagotonic and adrenergic tone is relevant to total HRV power and its various components in anxiety disorders (Cohen, Matar, Kaplan, \& Kotler, 1999; Friedman, 2007). Also, HRV measures have proved helpful to evaluate the effects of different psychiatric treatments, such as drugs, cognitive-behavioral therapy, and transcranial magnetic stimulation (Carney et al., 2000; Cohen, Kotler, Matar, \& Kaplan, 2000b; Fraguas et al., 2007; Middleton \& Ashby, 1995; Nishith et al., 2003; Siepmann, Ziemssen, Mueck-Weymann, Kirch, \& Siepmann, 2007; Udupa et al., 2007). With reference to EMDR treatment, the measures of HRV were employed to assess modifications within discrete sessions (Elofsson et al., 2008; Sack et al., 2008) and during a course of treatment (Sack et al., 2007) of PTSD patients.

\section{Purpose of the Study}

Our study was aimed at establishing the sensitivity to therapeutic change of selected symptom scales and HRV measures during the course of EMDR treatment of trauma-exposed clients, irrespective of the categorical diagnosis of PTSD. We hypothesized that the favorable effects on psychological symptom scales would be associated with an increase of the parasympathetic tone as measured by HRV recording.

\section{Methods}

\section{Psychopathological Assessment}

All of the following instruments were administered according to the standard Italian versions (Conti, 1999).

Impact of Event Scale (IES). The IES is a well validated and widely used 15 -item self-report questionnaire evaluating experiences of avoidance (IES AVOID) and intrusion (IES INTR) reflecting the 
overall intensity of posttraumatic stress reactions (IES TOTAL; Horowitz, Wilner, \& Alvarez, 1979; Sundin \& Horowitz, 2002).

90-Item Symptom Check List Revised (SCL-90-R). The SCL-90-R is a validated scale of psychiatric symptoms with nine primary symptom dimensions as well as three global indices of distress (Derogatis \& Cleary, 1977a, 1977b). Primary dimensions are the following: Anxiety (ANX), Phobic Anxiety (PHOB), Hostility (HOS), Depression (DEP), Paranoid Ideation (PAR), Obsessive-Compulsive (O-C), Psychoticism (PSY), Interpersonal Sensitivity (I-S), Somatization (SOM); the total indices include: Global Severity Index (GSI), Positive Symptom Distress Index (PSDI), Positive Symptom Total (PST).

Brief COPE Inventory (BCI). The BCI (Carver, 1997) is a shortened version of the original COPE consisting of 28 items, that is, two items for each of the 14 coping strategies that people may use when they meet difficult or stressful events.

Mini-International Neuropsychiatric Interview (MINI) ver. 5.0. The MINI (Sheehan et al., 1998) is a structured diagnostic interview evaluating various Axis I disorders of the DSM-IV-TR (APA, 2000), including major depressive episode, dysthymia, mania, hypomania, panic disorder, agoraphobia, social phobia, obsessive-compulsive disorder, PTSD, alcohol dependence or abuse, psychoactive substance dependence or abuse, anorexia, and bulimia.

Global Assessment of Functioning (GAF). The GAF is a 100-point tool rating the overall psychological, social and occupational functioning, as included in the DSM-IV-TR within the section on multiaxial assessments (APA, 2000).

\section{Physiological Assessment}

Patients underwent a 90-min. recording of Holter ECG, using 3-channel digital recorders monitoring the bipolar chest leads CM5, CM1, and modified aVF. The ECG recordings were analyzed by an expert cardiologist, who was unaware of the psychological data, using the Oxford Medilog Excel 3.0 device (Oxford, Abingdon, UK).

All the experimental procedures were carried out in a day-hospital quiet room without thermoregulatory distress. To minimize anticipatory anxiety, a pleasant atmosphere was maintained and a detailed explanation of the procedure was supplied.

HRV was assessed on Holter recording both in the time domain and in the frequency domain, after full ECG revision and editing of beats, when indicated. Time-domain HRV variables included the standard deviation of all R-R intervals (SDNN) and the square root of the mean of the sum of the squares of differences between adjacent R-R intervals (R-MSSD). The mean R-R interval (MEAN), which is inversely related to the heart beat rate, was also obtained for each recording.

In the frequency domain, HRV was assessed in the range of frequencies of $0-0.5 \mathrm{~Hz}$, by a fast Fourier transform spectral analysis algorithm, with a spectral resolution of $0.0005 \mathrm{~Hz}$, using the version 7.0 Oxford HRV analysis package 13. The amplitude of the following frequency-domain HRV variables was obtained: low frequency (LF; i.e., 0.04-0.15 Hz), which reflects both cardiac sympathetic and parasympathetic activity; and high frequency (HF; i.e., 0.15-0.40 $\mathrm{Hz}$ ), which mainly reflects cardiac parasympathetic activity. Furthermore, in order to obtain a measure of sympathovagal balance, the low frequency/high frequency ratio $(\mathrm{LF} / \mathrm{HF})$ was calculated.

All the HRV variables were assessed for separate intervals during each procedure of assessment: waiting (A), trauma recall (B), treatment (C) (see Figure 1).

\section{Participants}

Among the outpatients attending our clinic for anxiety disorders, eligible subjects were: (a) aged 18-65; (b) exposed to at least one small-t traumatic event; (c) experiencing psychopathological symptoms (IES score for each item $\geq 1$ ) and personal impairment (GAF score $\leq 70$ ) for at least 3 months. Participants were not assessed for PTSD symptom criteria because the traumatic event was a "small t" trauma. Subjects were excluded from the study if they suffered from comorbid psychosis, dementia, cardiac disease, or any somatic condition affecting the autonomic nervous system (e.g., diabetes mellitus, neurological disorders, etc.); moreover, subjects were excluded if they were currently being treated with psychotropic drugs (except low-dose hypnotic benzodiazepines) or

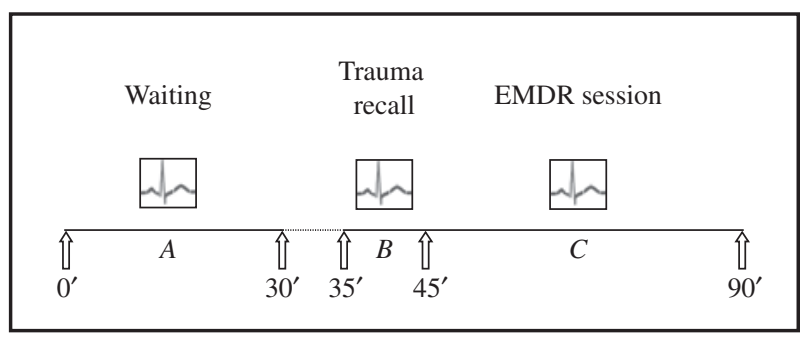

FIGURE 1. Intervals for HRV measures.

Note. Intervals $(A, B, C)$ and duration (in minutes) of the Holter ECG recording for HRV measures during each procedure of assessment. 
medications acting on the autonomic nervous system (e.g., $\beta$-blockers) or were currently street-drug users or tobacco smokers. Patients were not allowed to receive concurrent psychotherapeutic treatment and were excluded from the study in case of pending trauma-related lawsuit.

The study was approved by the local Medical Ethical Committee. After receiving information about the aims of the study, all participants gave their written consent.

\section{Study Design}

At the first appointment (assessment time: T0), patients underwent a baseline psychological assessment by means of MINI, IES, SCL-90-R, BCI. After this, the ECG Holter recording was started. The 90 min recording included:

- Interval A: 30 min waiting in the ward.

- Interval B: up to 10 min recalling the most salient aspects of the index trauma, while in a silent and comfortable room, with the narration audiotaped.

- Interval C: 45 min treatment session.

Assessments were conducted at the first and final sessions, and not during the intervening sessions. At the final session of treatment (T1) both symptom scales (IES, SCL-90-R) and physiological measures (HRV) were repeated using the same procedure as T0, except that during the interval B of ECG recording patients listened to their own audio-taped narration of the trauma, as recorded at T0, instead of recalling it. Follow-up evaluations were conducted after 30 (T2) and 90 days (T3) according to the same procedure as T1. In addition, the BCI questionnaire was administered again at $\mathrm{T} 3$.

\section{Treatment}

The EMDR treatment is aimed at processing traumatic memories. It consists of eight phases: (a) the therapist collects the traumatic history and identifies possible targets; (b) a therapeutic relationship is established and a "safe place" for visualization is chosen; (c) the sensory, cognitive, somatic, and affective components of the targeted memory are assessed; (d) the client is instructed to focus on the visual image, negative belief, and body sensations and to comply with the eye movements or other dual-attention stimuli; (e) when the patient can access the targeted memory without distress, the expression and consolidation of new cognitive insights are promoted while experiencing dual-attention stimulation; (f) the client is asked to notice any kind of bodily discomfort while focusing on the original image and on the positive cognition, in order to identify residual targets to be processed; $(\mathrm{g})$ the therapist determines whether the memory has been processed adequately and, if not, goes again into the "safe place" procedure; and (h) this reevaluation phase takes place at the beginning of every EMDR session following the initial one, to determine if the treatment gains from the previous session have been maintained (Shapiro \& Maxfield, 2002).

In our investigation the EMDR treatment consisted of 45-min individual sessions, and targeted memories associated with the index trauma identified at pretreatment evaluation. The EMDR technique followed the protocol suggested by Shapiro (2001) except for the sessions' duration, which was shortened considering the slight discomfort of wearing the recording device. The EMDR treatment was administered by two clinical psychologists, who had completed an EMDR Level II training. The treatment course (T0 to T1) was conducted on a weekly basis and all of the eight phases of the EMDR procedure were completed; the number of sessions ranged from a minimum of four to a maximum of six according to the individual response. The treatment was terminated when the participant rated the level of distress associated with the traumatic memory at $\leq 1$ on the Subjective Units of Distress (SUD) scale (Wolpe, 1969). During the follow-up session (T2 and T3) only the EMDR phases 5 to 8 were re-applied as necessary.

\section{Data Analysis and Statistics}

All data were entered into an SPSS database. An alpha of 0.05 was used for all statistical analyses. Given the small number of observations with a non-normal distribution of data, only nonparametric statistical tests were used.

The Friedman's test was applied to investigate changes in psychological and physiological measure across the four phases of treatment. Post hoc comparisons were carried out by Wilcoxon's paired test for variables showing significant changes at Friedman's test.

\section{Results}

\section{Sample Characteristics}

Participants in the study were four female outpatients seeking treatment for trauma-related disturbances at our Anxiety Disorders Unit. All patients had been exposed in adulthood to a relevant adverse event and suffered from persistent posttraumatic symptoms, 
although none of them fulfilled the entire set of DSM-IV-TR diagnostic criteria for PTSD. Adversities included: road accident without physical injury, wrong diagnosis of a fatal disease in a daughter, caring for a dying parent, and unexpected marital separation. The following were the main demographic characteristics: age $42.50( \pm 6.19)$ years; education 17.00 ( \pm 1.63$)$ years; married 2 out of 4 ; employed all except one.

No Axis I disorders were detected by the MINI interview. On the 15-item IES, the mean scores at baseline [Total score 51.00 ( \pm 4.24$)$; Intrusion 26.25 ( \pm 4.11$)$; Avoidance 24.75 ( \pm 2.22$)]$ were above the clinical cut-off for PTSD (Neal et al., 1994), indicating that our clients were significantly affected by posttraumatic symptoms. On the SCL-90-R, baseline scores [GSI 1.24 ( \pm 0.31$)$; PSDI 1.64 ( \pm 0.40$)$; PST 58.25 $( \pm 13.37)]$ were indicative of psychological distress and the need for treatment (Schauenburg \& Strack, 1999).

\section{Psychological and Physiological Correlates of Treatment}

In order to restrain valuable information from the large amount of collected data, we performed a preliminary aggregated analysis to point out measures showing a significant change across the EMDR treatment course (see Table 1).

Aggregated Data Analysis. The psychometric scores of the IES Total, Intrusion, and Avoidance scales changed significantly across the phases of treatment $(p \leq .05)$. Post hoc comparisons for each IES scale showed a borderline significant reduction $(p=$ .06) between T0 and T1, T0 and T2, T0 and T3; the Avoidance scale also showed a borderline significant reduction $(p=.06)$ between T1 and T3.

Only a few SCL-90-R indexes and subscales showed significant changes; these were Positive Symptom Total, Obsessive-Compulsive, and Psychoticism. Post hoc comparisons showed borderline significant reductions $(p=.06)$ for the Positive Symptom Total, Obsessive-Compulsive, and Psychoticism scales between $\mathrm{T} 0$ and $\mathrm{T} 1, \mathrm{~T} 0$ and $\mathrm{T} 2, \mathrm{~T} 0$ and $\mathrm{T} 3$; the Obsessive-Compulsive Scale also showed a borderline significant reduction $(p=.06)$ between $\mathrm{T} 1$ and T3.

Changes among HRV measures occurred for R-MSSD during the interval A, and for MEAN R-R, LF and LF/HF during the interval B, while no HRV measure changed significantly $(p \leq .05)$ during the interval C. Post hoc comparisons showed a borderline significant $(p=.06)$ increase for R-MSSD during the interval A between T0 and T3, T1 and T2, T1 and T3, T2 and T3; LF values during the interval B were reduced at a borderline significant level $(p=.06)$ between T0 and $\mathrm{T} 1, \mathrm{~T} 0$ and $\mathrm{T} 3$, T2 and T3; the LF/HF ratio during interval $\mathrm{B}$ diminished borderline significantly $(p=.06)$ between T0 and T1, T0 and T3; the MEAN $\mathrm{R}-\mathrm{R}$ during interval $\mathrm{B}$ increased (i.e., heart beat rate reduced) borderline significantly $(p=.06)$ between T0 and $\mathrm{T} 1, \mathrm{~T} 0$ and $\mathrm{T} 3, \mathrm{~T} 1$ and $\mathrm{T} 3, \mathrm{~T} 2$ and $\mathrm{T} 3$.

Finally, no statistically significant difference was observed in the measures of coping on the BCI, nor the Women Crime-related PTSD Scale of the SCL90-R (Saunders, Mandoki Arata, \& Kilpatrick, 1990).

Individual Observations. The foremost measures reaching a statistically significant modification across the phases of treatment are shown as individual observations in Table 1.

\section{Discussion}

The study compared symptom scores and HRV measures at the beginning and at the end of an EMDR treatment course (not evaluating intermediate sessions) and at 1- and 3-month follow-ups. Three intervals of ECG recordings were analyzed: waiting (interval A), trauma recall (interval B), and EMDR session (interval C).

Overall, symptom scores decreased between the baseline (T0) and the end of treatment (T1), with effects maintained throughout the follow-up (T2-T3). The decrease of psychological symptoms in our sample is consistent with the efficacy of EMDR treatment among PTSD patients (Högberg et al., 2008). It is also consistent with findings of Cvetek (2008) who found that EMDR reduced scores on the IES and distress during trauma recall for participants with small " $t$ " traumas. In our study a subjective benefit was clearly apparent, since both intrusive symptoms and general psychological distress were steadily reduced in selfrated measures after the treatment was completed.

As hypothesized, some HRV measures improved within different recording intervals (i.e., waiting and trauma recall) showing an increase of the parasympathetic tone, replicating the findings of Sack et al. (2007). This physiological change is indicative of arousal reduction. It suggests that after EMDR, the somatic load is decreased, enabling patients to deal with the environmental stimuli in a more adaptive way.

Taking into account the entire course of EMDR treatment (T0 to T1) and follow up (T2 and T3) the modifications of psychological and physiological measures differ in timing. Specifically during interval A of ECG recording (i.e., waiting for the treatment 
TABLE 1. Psychometric and Electrophysiological Modifications Across the Phases of Treatment

\begin{tabular}{|c|c|c|c|c|c|}
\hline & Measures & T0 & $\mathrm{T} 1$ & $\mathrm{~T} 2$ & T3 \\
\hline \multirow[t]{7}{*}{ Patient \#1 } & IES Total & 48 & 15 & 0 & 8 \\
\hline & IES Intrusion & 21 & 4 & 0 & 4 \\
\hline & IES Avoidance & 27 & 11 & 0 & 4 \\
\hline & R-MSSD (Interval A) & 22.8 & 21.2 & 25.8 & 27.4 \\
\hline & Mean R-R (Interval B) & 837.9 & 877.1 & 886.2 & 890.0 \\
\hline & LF (Interval B) & 37.4 & 36.8 & 53.6 & 29.9 \\
\hline & LF/HF (Interval B) & 2.8 & 2.6 & 2.7 & 1.86 \\
\hline \multirow[t]{7}{*}{ Patient \#2 } & IES Total & 51 & 33 & 29 & 11 \\
\hline & IES Intrusion & 27 & 14 & 11 & 4 \\
\hline & IES Avoidance & 24 & 19 & 18 & 7 \\
\hline & R-MSSD (Interval A) & 21.1 & 20.0 & 21.0 & 26.2 \\
\hline & Mean R-R (Interval B) & 920.2 & 972.0 & $1,014.3$ & $1,015.0$ \\
\hline & LF (Interval B) & 24.0 & 11.3 & 23.1 & 9.6 \\
\hline & LF/HF (Interval B) & 2.0 & 0.9 & 1.7 & 0.77 \\
\hline \multirow[t]{7}{*}{ Patient \#3 } & IES Total & 57 & 19 & 5 & 7 \\
\hline & IES Intrusion & 31 & 4 & 5 & 3 \\
\hline & IES Avoidance & 26 & 15 & 0 & 4 \\
\hline & R-MSSD (Interval A) & 25.4 & 14.0 & 20.9 & 46.5 \\
\hline & Mean R-R (Interval B) & 756.8 & 763.8 & 714.4 & 773.0 \\
\hline & LF (Interval B) & 44.0 & 13.6 & 19.2 & 18.6 \\
\hline & LF/HF (Interval B) & 2.0 & 0.8 & 2.3 & 1.26 \\
\hline \multirow[t]{7}{*}{ Patient \#4 } & IES Total & 48 & 5 & 16 & 7 \\
\hline & IES Intrusion & 26 & 2 & 10 & 7 \\
\hline & IES Avoidance & 22 & 3 & 6 & 0 \\
\hline & R-MSSD (Interval A) & 19.7 & 22.4 & 34.4 & 37.5 \\
\hline & Mean R-R (Interval B) & 540.0 & 736.5 & 829.4 & 836.0 \\
\hline & LF (Interval B) & 31.5 & 18.8 & 32.9 & 27.6 \\
\hline & LF/HF (Interval B) & 3.0 & 1.4 & 1.3 & 1.3 \\
\hline \multirow{7}{*}{$\begin{array}{l}\text { Total sample } \\
\qquad(M \pm S D)\end{array}$} & IES Total & $51 \pm 4.24$ & $18 \pm 11.6$ & $12.50 \pm 12.88$ & $8.25 \pm 1.89$ \\
\hline & IES Intrusion & $26.25 \pm 4.11$ & $6 \pm 5.42$ & $6.50 \pm 5.07$ & $4.50 \pm 1.73$ \\
\hline & IES Avoidance & $24.75 \pm 2.22$ & $12 \pm 6.83$ & $6 \pm 8.48$ & $3.75 \pm 2.87$ \\
\hline & R-MSSD (Interval A) & $22.25 \pm 2.45$ & $19.4 \pm 3.73$ & $25.52 \pm 6.34$ & $34.4 \pm 9.53$ \\
\hline & Mean R-R (Interval B) & $763.72 \pm 163.39$ & $837.35 \pm 108.46$ & $861.07 \pm 124.68$ & $878.35 \pm 102.67$ \\
\hline & LF (Interval B) & $34.22 \pm 8.52$ & $20.12 \pm 11.55$ & $32.2 \pm 15.39$ & $21.42 \pm 9.27$ \\
\hline & LF/HF (Interval B) & $2.45 \pm 0.53$ & $1.42 \pm 0.83$ & $2 \pm 0.62$ & $1.3 \pm 0.44$ \\
\hline
\end{tabular}

\footnotetext{
Note. T0 = beginning of treatment; $\mathrm{T} 1=$ end of the intensive phase of treatment; T2 = session of treatment 30 days after completing the intensive phase; T3 = session of treatment 90 days after completing the intensive phase.

*Only measures showing a statistically significant modification $(p \leq .05)$ are shown (see text).
}

session), the reduction in psychological symptoms chronologically preceded the change in HRV measures, as the IES scores lessened continuously from T0 to T3 while the R-MSSD measures improved from T2 to T3 only. On the other hand, when the interval B of ECG recording (i.e., trauma recall) is considered, psychological and physiological phenomena tended to co-occur, since HRV measures showed improvement at T1. This matches in part the results of Sack et al. (2007) who investigated the trauma script reactivity among PTSD patients. Of major interest, this observation suggests that participants' general level of physiological reactivity may improve as a function of memory reprocessing, in accordance with a "topdown" (or cognitive/affective) model of trauma processing instead of a "bottom-up" (or bodily/affective) one (Shapiro \& Maxfield, 2002; Stickgold, 2002).

Possible changes during individual stimulation sets of single treatment sessions were not caught by our study design, since this specific phase of the EMDR procedure was encompassed within measures of the broader 45-min interval C; such phenomena have been experimentally demonstrated by others (Elofsson et al., 2008; Sack et al., 2008). 
In sum, our results with a subclinical PTSD sample do not conflict with the accumulated knowledge regarding the treatment of full-blown PTSD by means of EMDR. An improvement was found with both psychological tests and HRV measures. We conclude that combined IES and HRV measures are appropriate to catch overt psychological and subtle physiological modifications during the treatment of disturbances that follow "small $t$ " traumatic exposures in female patients. Moreover, all the enrolled subjects underwent the full set of observations and completed the study procedures with no adverse effect. Since the research technique was sensitive, safe, and well accepted, further investigation is recommended.

First of all, these preliminary results should be confirmed in a larger sample including male subjects with randomization to some placebo psychological treatment (e.g., nonspecific counseling) and EMDR in order to investigate EMDR's efficacy in the treatment of minor trauma exposure.

We also recommend that future research investigate the responsiveness of nontraumatic Axis I disorders to EMDR as measured objectively by means of a psychosomatic method, using both physiological and psychological instruments. This method may provide a comprehensive evaluation of the participants' mind and body response to treatment.

Finally, we suggest that the procedure adopted in this study can be used to evaluate EMDR's mechanisms of action. Our research found that psychological changes chronologically preceded a group of physiological changes. Future studies may determine if the antecedence of psychological modifications during the treatment course and follow-up is a generalized phenomenon. Such findings should provide valuable insight into EMDR's mechanisms of action.

\section{References}

Agelink, M. W., Boz, C., Ullrich, H., \& Andrich, J. (2002). Relationship between major depression and heart rate variability: Clinical consequences and implications for antidepressive treatment. Psychiatry Research, 113, 139-149.

American Psychiatric Association. (2000). Diagnostic and statistical manual of mental disorders (4th ed., text rev.). Washington, DC: American Psychiatric Press.

Bisson, J. I., Ehlers, A., Matthews, R., Pilling, S., Richards, D., \& Turner, S. (2007). Psychological treatments for chronic post-traumatic stress disorder: Systematic review and meta-analysis. British Journal of Psychiatry, 190, 97-104.

Boettger, S., Hoyer, D., Falkenhahn, K., Kaatz, M., Yeragani, V. K., \& Bär, K. J. (2006). Altered diurnal autonomic variation and reduced vagal information flow in acute schizophrenia. Clinical Neurophysiology, 117, 2715-2722.

Buckley, T. C., \& Kaloupek, D. G. (2001). A meta-analytic examination of basal cardiovascular activity in posttraumatic stress disorder. Psychosomatic Medicine, 63, 585-594.

Carlson, J. G., Chemtob, C. M., Rusnak, K, Hedlund, N. L., \& Moraoka, M. Y. (1998). Eye movement desensitization and reprocessing (EMDR) treatment for combat-related posttraumatic stress disorder. Journal of Traumatic Stress, 11, 3-24.

Carney, R. M., Freedland, K. E., Stein, P. K., Skala, J. A., Hoffman, P., \& Jaffe, A. S. (2000). Change in heart rate and heart rate variability during treatment for depression in patients with coronary heart disease. Psychosomatic Medicine, 62, 639-647.

Carver, C. S. (1997). You want to measure coping but your protocol's too long: Consider the Brief COPE. International Journal of Behavioral Medicine, 4, 92-100.

Cohen, H., Benjamin, J., Geva, A. B., Matar, M. A., Kaplan, Z., \& Kotler, M. (2000a). Autonomic dysregulation in panic disorder and in post-traumatic stress disorder: Application of power spectrum analysis of heart rate variability at rest and in response to recollection of trauma or panic attacks. Psychiatry Research, 96, 1-13.

Cohen, H., Kotler, M., Matar, M., \& Kaplan, Z. (2000b). Normalization of heart rate variability in post-traumatic stress disorder patients following fluoxetine treatment: Preliminary results. Israel Medical Association Journal, 2, 296-301.

Cohen, H., Matar, M. A., Kaplan, Z., \& Kotler, M. (1999). Power spectral analysis of heart rate variability in psychiatry. Psychotherapy and Psychosomatics, 68, 59-66.

Conti, L. (1999). Repertorio delle Scale di Valutazione in Psichiatria. Firenze: Società Editrice Europea.

Cvetek, R. (2008). EMDR treatment of distressful experiences that fail to meet the criteria for PTSD. Journal of EMDR Practice and Research, 2, 2-14.

Derogatis, L. R., \& Cleary, P. A. (1977a). Confirmation of the dimensional structure of the SCL-90: A study in construct validation. Journal of Clinical Psychology, 33, 981-989.

Derogatis, L. R., \& Cleary, P. A. (1977b). Factorial invariance across gender for the primary symptom dimensions of the SCL-90-R. British Journal of Social and Clinical Psychology, 16, 347-356.

Elofsson, U. O., von Schèele, B., Theorell, T., \& Söndergaard, H. P. (2008). Physiological correlates of eye movement desensitization and reprocessing. Journal of Anxiety Disorders, 22, 622-634.

Fraguas, R., Jr., Marci, C., Fava, M., Iosifescu, D. V., Bankier, B., Loh, R., et al. (2007). Autonomic reactivity to induced emotion as potential predictor of response to antidepressant treatment. Psychiatry Research, 151, 169-172.

Friedman, B. H. (2007). An autonomic flexibility-neurovisceral integration model of anxiety and cardiac vagal tone. Biological Psychology, 74, 185-199. 
Friedman, B. H., \& Thayer, J. F. (1998). Anxiety and autonomic flexibility: A cardiovascular approach. Biological Psychology, 49, 303-323.

Gold, S. D., Marx, B. P., Soler-Baillo, J. M., \& Sloan, D. M. (2005). Is life stress more traumatic than traumatic stress? Journal of Anxiety Disorders, 19, 687-698.

Goldberger, A. L. (1991). Is the normal heartbeat chaotic or homeostatic? News in Physiological Sciences, 6, 87-91.

Högberg, G., Pagani, M., Sundin, O., Soares, J., AbergWistedt, A., Tärnell, B., et al. (2008). Treatment of post-traumatic stress disorder with eye movement desensitization and reprocessing: Outcome is stable in 35-month follow-up. Psychiatry Research, 15, 101-108.

Horowitz, M. J., Wilner, N., \& Alvarez, W. (1979). Impact of event scale: A measure of subjective stress. Psychosomatic Medicine, 41, 209-218.

Keane, T. M., Kolb, L. C., Kaloupek, D. G., Orr, S. P., Blanchard, E. B., Thomas, R. G., et al. (1998). Utility of psychophysiological measurement in the diagnosis of posttraumatic stress disorder: Results from a Department of Veterans Affairs Cooperative Study. Journal of Consulting and Clinical Psychology, 66, 914-923.

Middleton, H. C., \& Ashby, M. (1995). Clinical recovery from panic disorder is associated with evidence of changes in cardiovascular regulation. Acta Psychiatrica Scandinavica, 9, 108-113.

Mol, S. S., Arntz, A., Metsemakers, J. F., Dinant, G. J., Vilters-van Montfort, P. A., \& Knottnerus, J. A. (2005). Symptoms of post-traumatic stress disorder after nontraumatic events: Evidence from an open population study. British Journal of Psychiatry, 186, 494-499.

Montgomery, R. W., \& Ayllon, T. (1994). Eye movement desensitization across subjects: Subjective and physiological measures of treatment efficacy. Journal of Behavior Therapy and Experimental Psychiatry, 27, 219-229.

Neal, L. A., Bussuttil, W., Rollins, S., Herepath, R., Strike, P., \& Turnbull, G. (1994). Convergent validity of measures of Post-Traumatic Stress Disorder in a mixed military and civilian population. Journal of Traumatic Stress, 7, 447-455.

Nishith, P., Duntley, S. P., Domitrovich, P. P., Uhles, M. L., Cook, B. J., \& Stein, P. K. (2003). Effect of cognitive behavioral therapy on heart rate variability during REM sleep in female rape victims with PTSD. Journal of Traumatic Stress, 16, 247-250.

Orr, S. P., McNally, R. J., Rosen, G. M., \& Shalev, A. Y. (2004). Psychophysiologic reactivity: Implications for conceptualizing PTSD. In G. M. Rosen (Ed.), Posttraumatic stress disorder: Issues and controversies (pp. 101-126). Chichester: John Wiley \& Sons.

Orr, S. P., Metzger, L. J., Lasko, N. B., Macklin, M. L., Hu, F. B., Shalev, A. Y., et al. (2003). Physiologic responses to sudden, loud tones in monozygotic twins discordant for combat exposure: Association with posttraumatic stress disorder. Archives of General Psychiatry, 60, 283-288.
Orr, S. P., \& Roth, W. T. (2000). Psychophysiological assessment: Clinical applications for PTSD. Journal of Affective Disorders, 61, 225-240.

Pitman, R. K., Gilbertson, M. W., Gurvits, T. V., May, F. S., Lasko, N. B., Metzger, L. J., et al. (2006). Clarifying the origin of biological abnormalities in PTSD through the study of identical twins discordant for combat exposure. Annals of the New York Academy of Science, 1071, 242-254.

Pumprla, J., Howorka, K., Groves, D., Chester, M., \& Nolan, J. (2002). Functional assessment of heart rate variability: physiological basis and practical applications. International Journal of Cardiology, 84, 1-14.

Rogers, S., Silver, S. M., Goss, J., Obenchine, J., Willis, A., \& Whitney, R. L. (1999). A single session, group study of exposure and eye movement desensitization and reprocessing in treating posttraumatic stress disorder among Vietnam war veterans: Preliminary data. Journal of Anxiety Disorders, 13, 119-130.

Sack, M., Lempa, W., \& Lamprecht, F. (2007). Assessment of psychophysiological stress reactions during a traumatic reminder in patients treated with EMDR. Journal of EMDR Practice and Research, 1, 15-23.

Sack, M., Lempa, W., Steinmetz, A., Lamprecht, F., \& Hofmann, A. (2008). Alterations in autonomic tone during trauma exposure using eye movement desensitization and reprocessing (EMDR): Results of a preliminary investigation. Journal of Anxiety Disorders, 22, 1264-1271.

Saul, J. P. (1990). Beat-to-beat variations of heart rate reflect modulation of cardiac autonomic outflow. News in Physiological Sciences, 5, 32-37.

Saunders, B. E., Mandoki Arata, C., \& Kilpatrick, D. G. (1990). Development of a crime-related post-traumatic stress disorder scale for women within the Symptom Checklist-90-Revised. Journal of Traumatic Stress, 3, 439-448.

Schauenburg, H., \& Strack, M. (1999). Measuring psychotherapeutic change with the Symptom Checklist SCL 90 R. Psychotherapy and Psychosomatics, 68, 199-206.

Shapiro, F. (2001). Eye movement desensitization and reprocessing: Basic principles, protocols, and procedures (2nd ed.). New York: Guilford Press.

Shapiro, F., \& Maxfield, L. (2002). Eye movement desensitization and reprocessing (EMDR): Information processing in the treatment of trauma. Journal of Clinical Psychology, 58, 933-946.

Sheehan, D. V., Lecrubier, Y., Sheehan, K. H., Amorim, P., Janavs, J., Weiller, E., et al. (1998). The MiniInternational Neuropsychiatric Interview (MINI): The development and validation of a structured diagnostic psychiatric interview for DSM-IV and ICD-10. Journal of Clinical Psychiatry, 59(Suppl. 20), 22-33.

Siepmann, T., Ziemssen, T., Mueck-Weymann, M., Kirch, W., \& Siepmann, M. (2007). The effects of venlafaxine on autonomic functions in healthy volunteers. Journal of Clinical Psychopharmacology, 27, 687-691. 
Stein, P. K., Bosner, M. S., Kleiger, R. E., \& Conger, B. M. (1994). Heart rate variability: A measure of cardiac automic tone. American Heart Journal, 127, 1376-1381.

Stickgold, R. (2002). EMDR: A putative neurobiological mechanism of action. Journal of Clinical Psychology, 58, 61-75.

Sundin, E. C., \& Horowitz, M. J. (2002). Impact of Event Scale: Psychometric properties. British Journal of Psychiatry, 180, 205-209.

Udupa, K., Sathyaprabha, T. N., Thirthalli, J., Kishore, K. R., Raju, T. R., \& Gangadhar, B. N. (2007). Modulation of cardiac autonomic functions in patients with major depression treated with repetitive transcranial magnetic stimulation. Journal of Affective Disorders, 104, 231-236.

Van Hooff, M., McFarlane, A. C., Baur, J., Abraham, M., \& Barnes, D. J. (2009). The stressor Criterion-A1 and PTSD: A matter of opinion? Journal of Anxiety Disorders, 23, 77-86.

Wilhelm, F. H., \& Roth, W. T. (2001). The somatic symptom paradox in DSM-IV anxiety disorders: Suggestions for a clinical focus in psychophysiology. Biological Psychology, 57, 105-140.

Wilson, D. L., Silver, S. M., Covi, W. G., \& Foster, S. (1996). Eye movement desensitization and reprocessing: Effectiveness and autonomic correlates. Journal of Behavior Therapy and Experimental Psychiatry, 27, 219-229.

Wolpe, J. (1969). Practice of behavior therapy. New York: Pergamon Press.

Acknowledgments. This investigation was developed within the framework of the Ph.D. course in Research and Advanced Methodologies in Psychotherapy of the Catholic University of the Sacred Heart (Head: Prof. Giuseppe Ruggeri). Clinical procedures took place in the Day Hospital of Clinical Psychiatry of the "Agostino Gemelli” University Hospital (Chief: Prof. Pietro Bria). Treatment procedures were supported by the Italian EMDR Association, by reviewing the fidelity of EMDR protocol used with the sample. The EMDR treatment was administered by the trained psychologists Dr. Anna Rita Verardo and Dr. Renzo Barbato. Dr. Cristina Aurigemma, Dr. Lucy Barone, and Dr. Francesca Di Clemente from the Institute of Cardiology of the Catholic University of the Sacred Heart assisted the Authors in Holter ECG recordings and HRV analyses.

Correspondence regarding this article should be directed to Gino Pozzi, Anxiety Disorders Unit, Day Hospital of Clinical Psychiatry, Policlinico Universitario "Agostino Gemelli," 00168, Rome, Italy. E-mail: gpozzi@rm.unicatt.it. 